\title{
ICTIOFAUNA, RECURSOS ALIMENTARES E RELAÇÕES COM AS INTERFERÊNCIAS ANTRÓPICAS EM UM RIACHO URBANO NO SUL DO BRASIL
}

\author{
Deise Cristiane de Oliveira ${ }^{1}$ \& Sirlei Terezinha Bennemann ${ }^{2}$ \\ Biota Neotropica v5 (n1) - http://www.biotaneotropica.org.br/v5n1/pt/abstract?article+BN02905012005 \\ Recebido em 01/09/04 \\ Versão revisada recebida em 20/01/05. \\ Publicado em 01/02/05
${ }^{1}$ Curso de Pós-graduação em Ciências Biológicas (Mestrado), Departamento de Biologia Animal e Vegetal, Centro de Ciências Biológicas, Universidade Estadual de Londrina. 86051-970 - Londrina, PR - Brasil. deisecristiane@yahoo.com.br 970 - Londrina, PR - Brasil. sirlei@uel.br \\ ${ }^{2}$ Departamento de Biologia Animal e Vegetal, Centro de Ciências Biológicas, Universidade Estadual de Londrina. 86051-
}

\begin{abstract}
This research was performed in the upper portion of ribeirão Cambé, a Tibagi river subafluent, located at the urban area of Londrina, Paraná. The goals of this research were assessing qualitative and quantitative variations of fish species and of the food resources consumed by them related to environmental alterations. Four samples were taken in each of the five points (P1 to P5) selected along the stream, from November 2001 to August 2002. The number of species and diversity of food resources were different in each sampling point, with a direct relation between diversity of food resources and fish species. Detritus was the most abundant resource used by fishes in all sampling points except in P1, where insects and terrestrial plants were among the abundant food item. In P2, P3, and P4, the abundance of detritus consumed by fishes is related to the abundance of Poecilia reticulata, highly tolerant to antropic impacts. In P5, P. reticulata e Phalloceros caudimaculatus foraged basically on detritus, whereas four species of Tetragonopterinae that preyed mainly upon insects and plants behaved as generalists. Along the studied portion of ribeirão Cambé, the effects of antropic impacts typical of urbanized areas such as removal of riparian forest, effluent discharges, channel alteration, and introduction of exotic or allochothonus species were evident. Furthermore, many species behaved as specialists consuming an abundant item (detritus) instead of a wider array of food resources as expected in undegraded streams.
\end{abstract}

Key words: Fishes, urban stream, food resources, environmental alterations.

\section{Resumo}

Esta pesquisa foi desenvolvida na porção superior do ribeirão Cambé, subafluente do rio Tibagi, área urbana de Londrina, Paraná. O objetivo foi verificar as variações qualitativas e quantitativas das espécies de peixes e dos recursos alimentares consumidos pelas mesmas e suas relações com as alterações ambientais. Foram realizadas quatro coletas em cada um dos cinco trechos (P1 a P5), durante o período de novembro de 2001 a agosto 2002. Os resultados foram distintos em cada ponto em número de espécies e diversidade de itens alimentares, havendo correspondência entre ambos. Um menor número de espécies de peixes foi encontrado onde a diversidade de itens alimentares foi menor. Detrito foi o item alimentar mais abundante utilizado pelos peixes em todos os pontos, exceto em P1, onde insetos e vegetais terrestres estiveram também entre os alimentos abundantes. Em P2, P3 e P4 o alto valor percentual do peso de detrito consumido está relacionado com a abundância de Poecilia reticulata que é altamente tolerante às alterações antrópicas. Em P5, P. reticulata e Phalloceros caudimaculatus foram responsáveis pela quantidade de detrito consumido, enquanto restos de insetos e vegetais foram utilizados pelas quatro espécies de Tetragonopterinae, que se comportaram como generalistas. No ribeirão Cambé, ficou evidente o efeito de alterações antrópicas típicas de ambiente urbano, como a remoção da mata ciliar, lançamento de efluentes na água, mudança do canal e a introdução de espécies exóticas ou alóctones, entre as principais. Além disso, muitas espécies se comportaram como especialistas utilizando um alimento abundante (detrito) e, não uma variedade de recursos como seria esperado em riachos menos degradados.

Palavras-chave: Peixes, riacho urbano, recursos alimentares, alterações ambientais. 


\section{Introdução}

Nos últimos anos os estudos de riachos têm se intensificado, pois suas dimensões reduzidas tornam estes ambientes mais sensíveis à ação humana. Na bacia do rio Paraná particularmente, essas fontes hídricas se encontram em avançado estado de degradação antes mesmo de serem estudadas (Luiz et al. 1998). Para a compreensão destes ecossistemas um dos caminhos pode ser o estudo da utilização dos alimentos pelas espécies de peixes, pois as informações disponíveis são ainda escassas, principalmente quanto à origem das fontes de alimentos e as relações com as áreas adjacentes.

A presença de organismos sensíveis a alterações antropogênicas é uma condição freqüentemente observada em ambientes considerados menos alterados (Araújo 1998). De acordo com Lyons et al. (1995), os riachos com boas condições de integridade possuem espécies de peixes nativas com várias classes de tamanhos e a estrutura trófica é balanceada. À medida que a influência antrópica aumenta, as espécies mais sensíveis começam a desaparecer e a estrutura trófica é alterada.

Os riachos subafluentes do rio Tibagi na bacia do Alto Paraná têm sido estudados com enfoque em diversas áreas, tais como diversidade (Shibatta et al. 2002), o tamanho dos peixes (Shibatta \& Cheida 2003), e reprodução (Veregue \& Orsi 2003).

O ribeirão Cambé, um subafluente do rio Tibagi, na sua porção superior encontra-se totalmente inserido na área urbana da cidade de Londrina, com diferentes alterações nesta extensão. Por tratar-se da porção superior da bacia, seria esperado encontrar um número e composição de espécies de peixes e recursos alimentares semelhante em cada trecho desta extensão, no entanto, a nossa hipótese é que serão encontradas distintas composições de espécies e recursos alimentares em diferentes trechos, pois cada um é afetado por diferentes tipos impactos. Assim, nosso objetivo foi verificar as variações qualitativas e quantitativas das espécies de peixes e dos recursos alimentares consumidos pelas mesmas, em cinco pontos de coleta na porção superior do ribeirão Cambé. Três aspectos foram analisados em cada trecho: a) a composição da ictiofauna; b) os itens alimentares consumidos pelas espécies; e c) as relações dos possíveis impactos antrópicos sobre estes dois parâmetros.

\section{Material e Métodos}

\section{1 Área de estudo}

O estudo foi realizado no ribeirão Cambé, um subafluente do rio Tibagi, que nasce no município de Cambé e deságua no ribeirão Três Bocas, no município de Londrina. As amostragens foram desenvolvidas no curso superior do ribeirão Cambé, onde foram estabelecidos cinco pontos (P1 a P5) ao longo de 8 km de extensão. As coletas ocorreram em freqüência trimestral, em cada ponto entre novembro de 2001 e agosto de 2002. A localização e características de cada ponto estão apresentadas na Tabela 1 e Figura 1.

Em cada ponto, foi padronizado um trecho de $50 \mathrm{~m}$ de extensão delimitado com redes de tela (malha $2 \mathrm{~mm}$ ). Os peixes foram coletados com tarrafas (malha $2 \mathrm{~cm}$ entre nós) peneiras (malha $2 \mathrm{~mm}$ ) e redes de arrasto (malha $2 \mathrm{~mm}$ ).

\subsection{Processamento do material biológico}

Os peixes coletados foram fixados em solução de formalina a 10\% e conservados em álcool 70\%. Os espécimes foram identificados conforme literatura apresentada em Shibatta et al. (2002) e o material testemunho se encontra depositado no Museu de Zoologia da Universidade Estadual de Londrina.

Após obtenção de dados biométricos e peso dos exemplares, os estômagos foram retirados para a análise do conteúdo estomacal. Para obtenção do peso total do conteúdo alimentar, cada estômago foi pesado em balança semi-analítica, antes e após a remoção do conteúdo. Os itens foram identificados sob microscópio estereoscópico seguindo os manuais de identificação de Stehr (1987) e Pérez (1998), e analisados pelos métodos de freqüência de ocorrência e gravimétrico descritos por Hyslop (1980). O segundo método foi modificado, e o peso de cada item nos estômagos que continham mais de um, foi calculado a partir de uma regra de três simples, com base na proporção relativa do item, estimada visualmente.

\subsection{Análise de dados}

Para análise dos itens alimentares foram realizadas comparações qualitativa e quantitativa dos percentuais de ocorrência e dos pesos por espécie e no total dos peixes, em cada trecho de coleta.

Para a interpretação dos resultados foi realizada uma análise de similaridade utilizando o índice quantitativo de Bray-Curtis entre grupos, levando em consideração os percentuais dos pesos dos itens consumidos pelos peixes em cada ponto amostrado. Os valores obtidos foram colocados em uma matriz de similaridade, para posterior aplicação de Análise de Cluster, com agrupamento em pares, utilizando o Programa Past, com variação de 0 a 1.

Outro método empregado foi a análise gráfica de Costello (1990), utilizando a distribuição dos percentuais de ocorrência e peso de cada um dos itens alimentares, em cada ponto. As duas diagonais do gráfico representam a importância dos itens consumidos pelos peixes em cada um dos pontos. Os alimentos dominantes foram aqueles cujos pontos estiveram distribuídos acima de 50\% de ocorrência e do peso. 


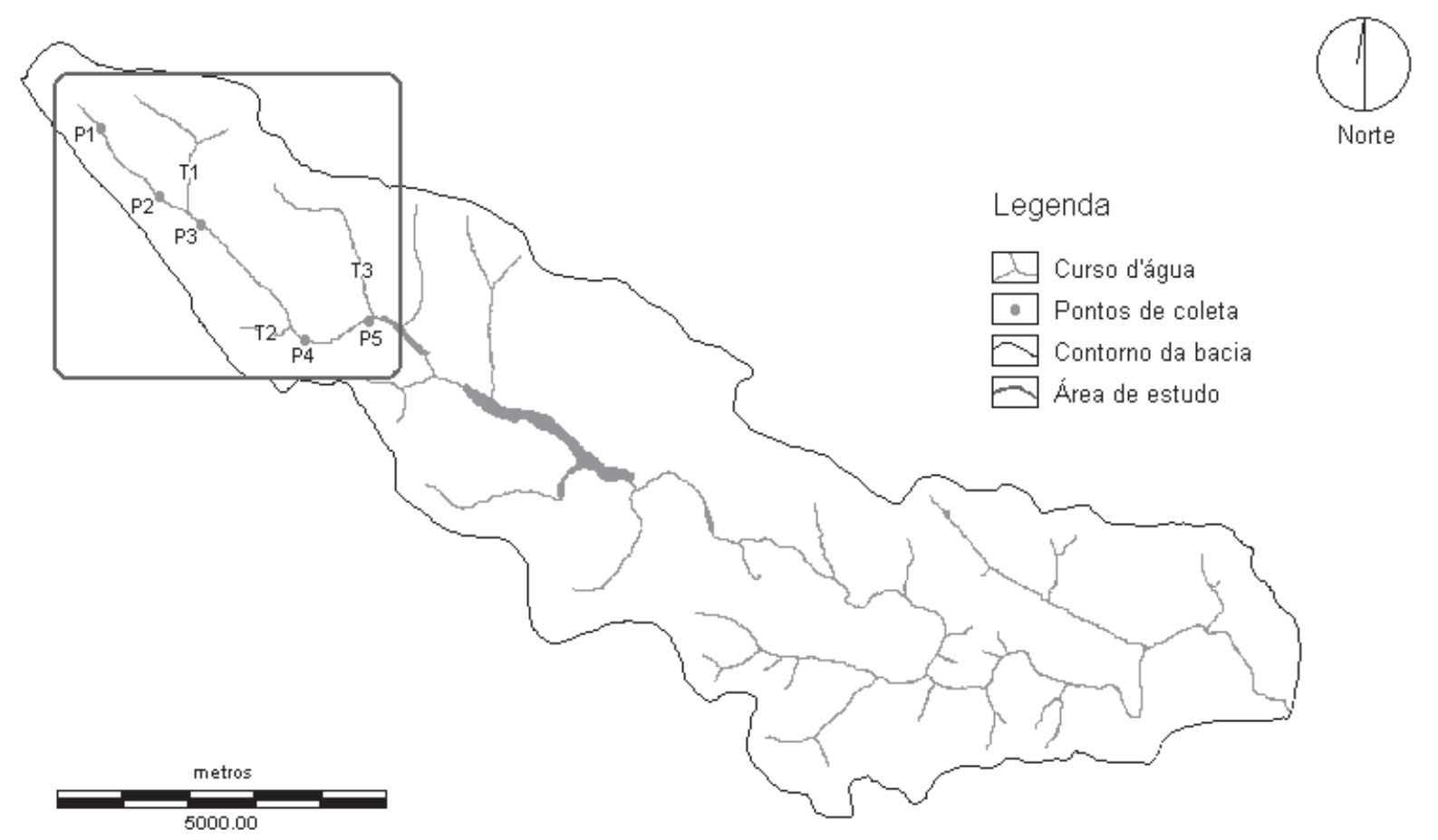

Figura 1. Bacia do Alto ribeirão Cambé, na área urbana da cidade de Londrina. Os tributários estão representados por T1 (Córrego Cacique), T2 (Córrego da Mata) e T3 (Córrego Baroré). Fonte: Laboratório de Biodiversidade e Restauração de Ecossistemas/UEL, 2002 (modificado).

Tabela 1. Características ambientais dos pontos de coleta da bacia do ribeirão Cambé.

\begin{tabular}{|c|c|c|c|c|c|}
\hline & Ponto 1 & Ponto 2 & Ponto 3 & Ponto 4 & Ponto 5 \\
\hline Coordenadas & $\begin{array}{c}23^{\circ} 17^{\prime} 15^{\prime \prime S} \\
51^{\circ} 13^{\prime} 58^{\prime \prime} \mathrm{W}\end{array}$ & $\begin{array}{c}23^{\circ} 17^{\prime} 49^{\prime \prime S} \\
51^{\circ} 13^{\prime} 27^{\prime \prime} \mathrm{W}\end{array}$ & $\begin{array}{l}23^{\circ} 18^{\prime} 06^{\prime \prime} \mathrm{S} \\
51^{\circ} 13^{\prime} 05^{\prime \prime} \mathrm{W}\end{array}$ & $\begin{array}{l}23^{\circ} 18^{\prime} 45^{\prime \prime} \mathrm{S} \\
51^{\circ} 12^{\prime} 17^{\prime \prime} \mathrm{W}\end{array}$ & $\begin{array}{c}23^{\circ} 19^{\prime} 11^{\prime \prime S} \\
51^{\circ} 11^{\prime} 47^{\prime \prime} \mathrm{W}\end{array}$ \\
\hline $\begin{array}{l}\text { Largura média } \\
(\mathrm{cm})\end{array}$ & 144,1 & 141,0 & 291,2 & 346,3 & 918,8 \\
\hline $\begin{array}{l}\text { Profundidade } \\
\text { média }(\mathrm{cm})\end{array}$ & 48,1 & 37,1 & 28,8 & 36,8 & 63,2 \\
\hline Substrato & $\begin{array}{l}\text { Argiloso, com } \\
\text { rochas }\end{array}$ & $\begin{array}{l}\text { Argiloso, com } \\
\text { cascalho e } \\
\text { pedras grandes }\end{array}$ & $\begin{array}{l}\text { Cascalho, com } \\
\text { áreas argilosas }\end{array}$ & rochoso & $\begin{array}{l}\text { Rochoso no } \\
\text { poção e argiloso } \\
\text { no restante da } \\
\text { área }\end{array}$ \\
\hline $\begin{array}{l}\text { Vegetação } \\
\text { marginal }\end{array}$ & $\begin{array}{l}\text { Taboas (Typha } \\
\text { cf. dominguensi) }\end{array}$ & $\begin{array}{l}\text { Gramíneas altas } \\
\text { e árvores }\end{array}$ & $\begin{array}{c}\text { Árvores e } \\
\text { plantas rasteiras } \\
\text { (gramíneas e } \\
\text { herbáceas) }\end{array}$ & $\begin{array}{l}\text { Mata mais } \\
\text { preservada }\end{array}$ & $\begin{array}{c}\text { Árvores nativas } \\
\text { e gramíneas } \\
\text { altas }\end{array}$ \\
\hline Impactos & $\begin{array}{c}\text { Entroncamento } \\
\text { de rodovias, } \\
\text { barramento à } \\
\text { jusante com } \\
\text { formação de } \\
\text { dois lagos }\end{array}$ & $\begin{array}{c}\text { Logo abaixo dos } \\
\text { dois lagos, área } \\
\text { marginal direita } \\
\text { com hortas. } \\
\text { Canalização. } \\
\text { Estrada } \\
\end{array}$ & $\begin{array}{l}\text { Pastagens. } \\
\text { Trânsito } \\
\text { constante de } \\
\text { animais e } \\
\text { pessoas }\end{array}$ & $\begin{array}{c}\text { Indústria de } \\
\text { empacotamento } \\
\text { de leite a } \\
\text { montante. }\end{array}$ & $\begin{array}{l}\text { Atividades de } \\
\text { lazer, pesca, } \\
\text { trânsito de } \\
\text { pessoas. Canal } \\
\text { modificado }\end{array}$ \\
\hline
\end{tabular}




\section{Resultados}

\subsection{Composição da ictiofauna}

Foram coletadas 15 espécies de peixes, num total de 5454 exemplares (Tabela 2), sendo que as mais abundantes foram Poecilia reticulata e Phalloceros caudimaculatus. As espécies $P$. reticulata, $P$. caudimaculatus, Bryconamericus iheringii, Tilapia rendalli e Astyanax scabripinnis representaram $86,9 \%$ do número total de exemplares coletados (Tabela 2). O tamanho dos exemplares analisados variou de 1,3 a 10,5 cm de comprimento padrão.

\subsection{Itens alimentares consumidos pelas espécies de peixes}

Para identificar os itens alimentares utilizados pelas espécies de peixes, foram analisados 1076 exemplares. No total foram identificados 30 tipos de itens alimentares, sendo que em P5 foi registrado o maior número (28) e em P4 o menor (17). Os valores dos pesos dos itens alimentares identificados em cada ponto amostrado constam na Tabela 3.

Tabela 2. Número de espécies e número de exemplares coletados e analisados (entre parênteses) nos cinco pontos de coleta.

\begin{tabular}{lccccc}
\hline Espécies & Ponto 1 & Ponto 2 & Ponto 3 & Ponto 4 & Ponto 5 \\
\hline $\begin{array}{l}\text { 1. Astyanax altiparanae } \\
\text { 2. Astyanax scabripinnis }\end{array}$ & $201(117)$ & $32(27)$ & $46(42)$ & $16(13)$ & $196(52)$ \\
$\begin{array}{l}\text { 3. Bryconamericus iheringii } \\
\text { 4. Callichthys callichthys }\end{array}$ & & $1(1)$ & $1(1)$ & & $506(115)$ \\
$\begin{array}{l}\text { 5. Geophagus brasiliensis } \\
\text { 6. Gymnotus carapo }\end{array}$ & $129(35)$ & $36(30)$ & & $59(13)$ \\
$\begin{array}{l}\text { 7. Hyphessobrycon cf. anisitsi } \\
\text { 8. Hypostomus ancistroides }\end{array}$ & $2(1)$ & $70(8)$ & $17(3)$ & & 1 \\
9. Oreochromis niloticus & & $64(41)$ & $103(41)$ & & $95(36)$ \\
$\begin{array}{l}\text { 10. Phalloceros caudimaculatus } \\
\text { 11. Poecilia reticulata }\end{array}$ & $540(116)$ & $6(2)$ & $5(2)$ & & 4 \\
12. Rhamdia quelen & $12(8)$ & $191(30)$ & $979(60)$ & $166(70)$ & $989(60)$ \\
13. Serrapinnus notomelas & & $5(3)$ & $2(1)$ & $3(2)$ & 2 \\
14. Synbranchus marmoratus & $3(1)$ & & & & $5(5)$ \\
15. Tilapia rendalli & & $386(40)$ & $89(24)$ & & $19(8)$ \\
Total de exemplares coletados & $\mathbf{7 5 8}$ & $\mathbf{8 8 5}$ & $\mathbf{1 3 8 5}$ & $\mathbf{1 8 5}$ & $\mathbf{2 2 4 1}$ \\
\hline Número de espécies & $\mathbf{5}$ & $\mathbf{1 0}$ & $\mathbf{1 2}$ & $\mathbf{3}$ & $\mathbf{1 2}$ \\
\hline
\end{tabular}

Em cada um dos pontos amostrados, observou-se grande variação no número de espécies (de 3 a 12) (Tabela 2). Em P1 a espécie nativa $P$. caudimaculatus ocorreu em maior número de indivíduos seguida por $A$. scabripinnis também nativa. A segunda espécie foi coletada em todos os trechos, mas somente em P1 foi abundante. Nos demais pontos, duas espécies introduzidas foram representadas por maior número de indivíduos: T. rendalli em P2 e P. reticulata em P3 a P5.
A diversidade e o peso dos itens alimentares consumidos foi variável entre os pontos, porém, 10 ocorreram nos cinco locais amostrados: restos vegetais, Coleoptera, Hemiptera, Hymenoptera, Ceratopogonidae, Chironomidae, outros Diptera, restos de insetos, restos de peixes e detritos (Tabela 3). 
Tabela 3. Abundância (peso em gramas) dos recursos alimentares totais, utilizados pelas espécies em cada trecho.

\begin{tabular}{|c|c|c|c|c|c|}
\hline$\underline{\text { Recursos }} \underline{\text { Alimentares }}$ & Ponto 1 & Ponto 2 & Ponto 3 & Ponto 4 & Ponto 5 \\
\hline \multicolumn{6}{|l|}{ I. Vegetais } \\
\hline 1) Gramíneas (sementes e folhas) & 0,957 & 0,581 & 0,613 & & 0,089 \\
\hline 2) Outras sementes & & & & & 0,134 \\
\hline 3) Plantas Aquáticas & & & & & 0,322 \\
\hline 4) Algas Filamentosas & & 0,13 & 0,003 & & 0,43 \\
\hline 5) Outras Algas & & & & & 0,024 \\
\hline 6) Restos Vegetais & 1,480 & 1,461 & 0,837 & 0,011 & 1,297 \\
\hline \multicolumn{6}{|l|}{ II. Insetos } \\
\hline 7) Coleoptera ${ }^{1}$ & 0,869 & 0,234 & 0,046 & 0,089 & 0,038 \\
\hline 8) Ephemeroptera² & 0,321 & 0,006 & & 0,01 & 0,013 \\
\hline 9) Hemiptera & 0,137 & 0,103 & 0,016 & 0,003 & 0,003 \\
\hline 10) Hymenoptera & 0,548 & 0,371 & 0,296 & 0,022 & 0,213 \\
\hline 11) Lepidoptera ${ }^{2}$ & 0,162 & & & & \\
\hline 12) Odonata ${ }^{2}$ & 0,003 & 0,071 & & & 0,005 \\
\hline 13) Trichoptera ${ }^{2}$ & & 0,006 & 0,001 & & 0,02 \\
\hline \multicolumn{6}{|l|}{ DIPTERA } \\
\hline 14) Ceratopogonidae & 0,003 & 0,081 & 0,073 & 0,003 & 0,032 \\
\hline 15) Chironomidae & 0,046 & 0,507 & 0,094 & 0,136 & 0,302 \\
\hline 16) Culicidae & 0,051 & & & & \\
\hline 17) Muscidae & 0,022 & & & 0,003 & 0,005 \\
\hline 18) Simuliidae & & 0,044 & 0,004 & 0,025 & 0,014 \\
\hline 19) Outros Diptera & 0,240 & 0,149 & 0,019 & 0,041 & 0,215 \\
\hline 20) Restos de insetos ${ }^{3}$ & 0,804 & 0,223 & 0,281 & 0,221 & 0,975 \\
\hline \multicolumn{6}{|l|}{ III. Crustáceos } \\
\hline 21) Isopoda & 0,170 & & 0,003 & & 0,001 \\
\hline 22) Microcrustáceos ${ }^{5}$ & & 0,053 & 0,016 & 0,003 & 0,008 \\
\hline \multicolumn{6}{|l|}{ IV. Peixes } \\
\hline 23) Escamas e restos & 0,014 & 0,033 & 0,313 & 0,003 & 0,025 \\
\hline \multicolumn{6}{|l|}{ V. Diversos Grupos } \\
\hline 24) Annelida & & 0,02 & 0,246 & 0,039 & 0,036 \\
\hline 25) Bryozoa & & 0,001 & 0,006 & & 0,418 \\
\hline 26) Diversos Táxons ${ }^{4}$ & 0,092 & 0,006 & 0,003 & & 0,199 \\
\hline 27) Testacealobosia & 0,025 & & 0,001 & & 0,002 \\
\hline \multicolumn{6}{|l|}{ VI. Outros } \\
\hline 28) Detritos & 1,519 & 5,291 & 3,501 & 0,708 & 2,977 \\
\hline 29) Sedimentos & 0,323 & 0,403 & 0,602 & 0,11 & 0,182 \\
\hline 30) Restos orgânicos & & 0,279 & 0,037 & 0,041 & 0,546 \\
\hline Total & 7,786 & 10,053 & 7,011 & 1,468 & 8,525 \\
\hline $\mathrm{N}^{0}$ de recursos alimentares & 20 & 22 & 22 & 17 & 28 \\
\hline $\mathbf{N}^{0}$ de exemplares analisados & 243 & 188 & 207 & 85 & 353 \\
\hline
\end{tabular}

(1)maioria adultos; (2) imaturos; (3) Não identificados e os que tiveram rara ocorrência; (4) Acari, Rotifera, Nematoda, Diplopoda; (5) Cladocera, Copepoda e Ostracoda 
Em P1 (Figura 2A) a maior proporção de itens ingeridos foi de origem animal, enquanto nos demais pontos a categoria de detrito/sedimento/restos orgânicos foi a que representou a maior proporção. Em P4 os recursos na categoria vegetal foram pouco significativos (menos que 1\%). Quanto à origem, as proporções dos recursos consumidos foram semelhantes em P2 a P5, com maiores valores encontrados para a categoria mista (Figura 2B). Em P1, diferentemente dos demais, os recursos de origem alóctone foram predominantes.
Quatro níveis de agrupamento foram reconhecidos através dos percentuais dos pesos dos itens alimentares em cada ponto (Figura 3). Detrito foi o item consumido em maior quantidade pelos peixes, e foi o principal responsável pelos altos valores de similaridade entre os pontos amostrados. Contudo, no caso de P2 e P3, as semelhanças não se restringiram somente à quantidade de detritos (53\% e 50\% do total consumido, respectivamente), mas também à similaridade entre os demais itens, com valores superiores a 80\%. Entre P1 e P5, os mais dissimilares, foi registrado o

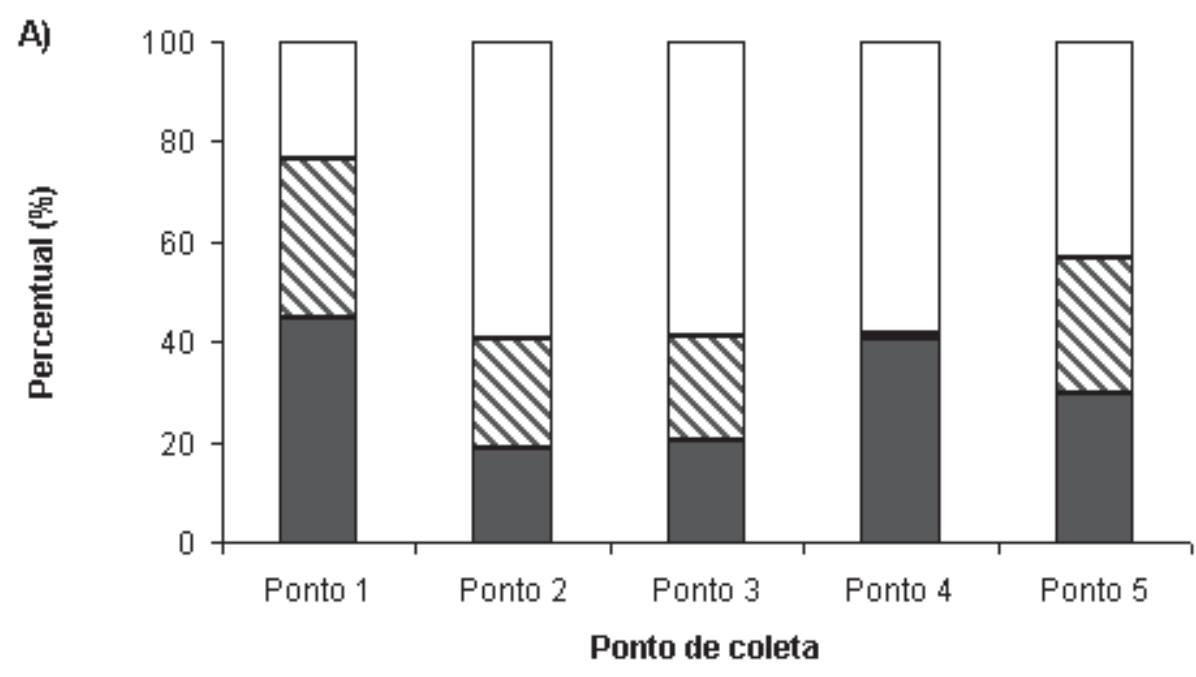

$\square$ Animal $\otimes$ Vegetal $\square$ Detisedir.orgân

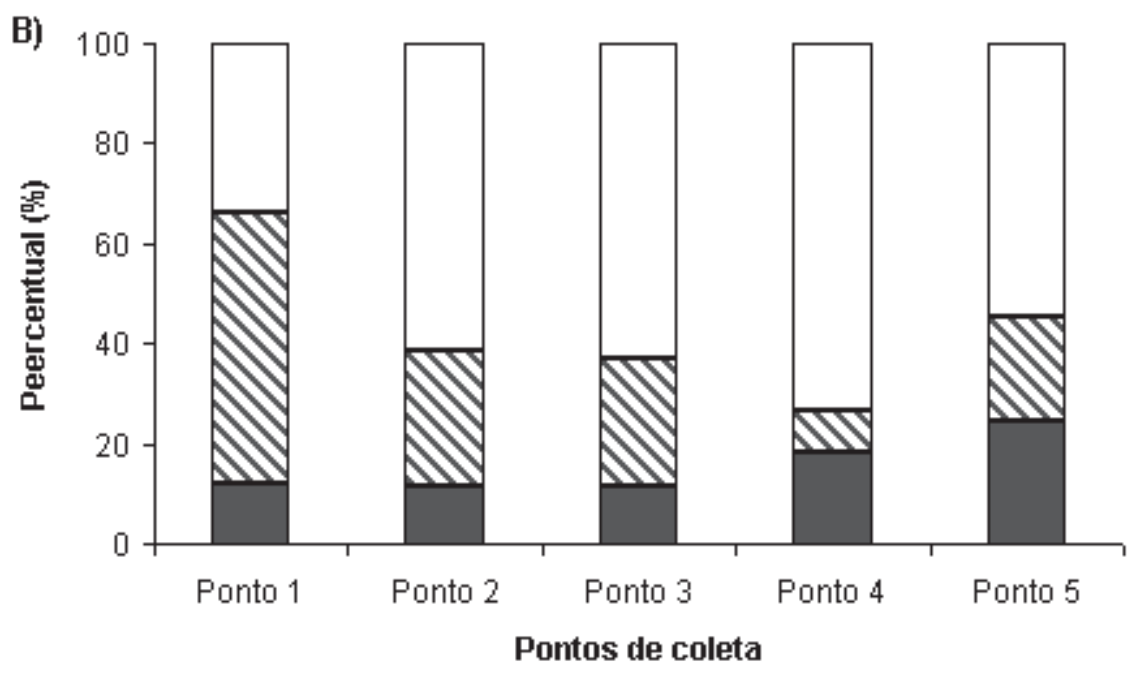

$\square$ Autóctone $\triangle$ Alóctone $\square$ Misto

Figura 2. Percentuais dos pesos dos itens alimentares consumidos pelas espécies de peixes em cada ponto. A) categorias: animal, vegetal e detrito/sedimento/restos orgânicos; B) origem: autóctone, alóctone e misto. 
consumo de um maior número de itens, dos quais restos de insetos e restos vegetais estiveram entre os mais consumidos. Em P5, o percentual de detrito foi de 35\%, e em P1, 20\%. Nestes dois pontos não foi verificada uma dominância de um único item alimentar.
Hypostomus ancistroides, $P$. reticulata e T. rendalli (Tabela 4) que foram as espécies mais abundantes. Os demais itens representados na Figura 4 (gramíneas, restos vegetais, Chironomidae, Hymenoptera e restos de insetos) foram

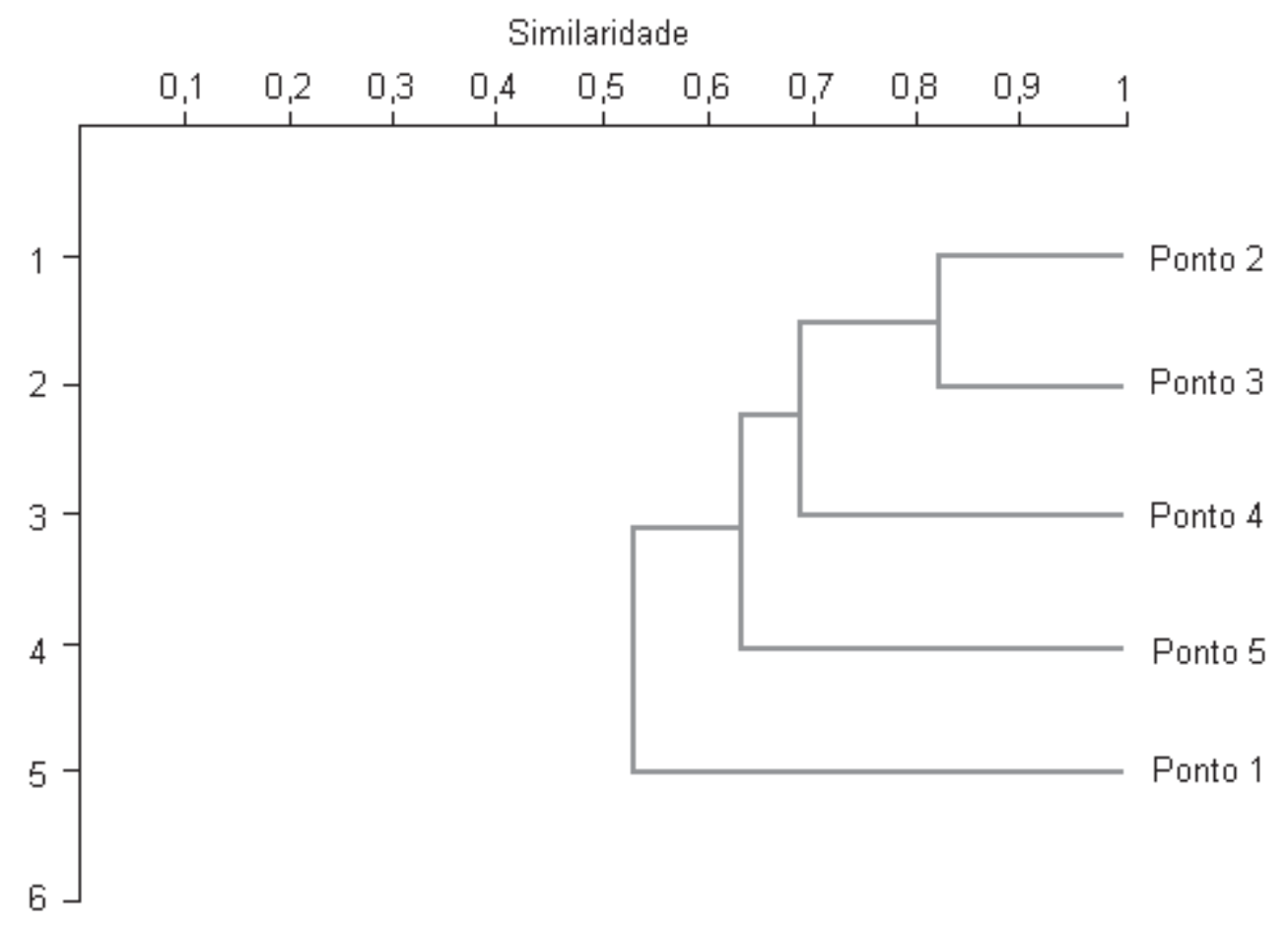

Figura 3. Dendrograma de similaridade utilizando o coeficiente de Bray-Curtis entre os pontos de coleta, considerando os percentuais do peso dos itens alimentares consumidos pelos peixes.

\subsection{Relações entre as espécies de peixes e os itens alimentares consumidos}

Apesar da relativa diversidade de itens ingeridos pelos peixes, 10 foram os mais importantes em ocorrência e/ ou peso. No entanto, nota-se que detrito foi o mais consumido, tanto em ocorrência quanto em peso, em todos os pontos, exceto em P1 (Figura 4). Neste ponto, detrito e restos vegetais tiveram proporções similares em peso. A espécie onívora $A$. scabripinnis foi a responsável por $76 \%$ do total (em peso) dos itens consumidos pelos peixes neste ponto. Os itens gramíneas, Coleoptera, Hymenoptera, restos vegetais e restos de insetos, representados na Figura 4, foram consumidos principalmente por esta espécie (Tabela 4). O detrito, neste ponto, foi consumido essencialmente por $P$. caudimaculatus (Tabela 4).

Nos outros pontos os elevados percentuais de ocorrência e peso do detrito representados na Figura 4, são explicados pela maior quantidade de espécies e indivíduos que se comportaram como detritívoros. Em P2 e P3 este item foi alimento principal para Geophagus brasiliensis, consumidos em maior quantidade por A. scabripinnis, no entanto as espécies anteriormente citadas também utilizaram estes recursos, porém em menores quantidades que detrito (Tabela 4).

Em P4 P. reticulata foi a espécie dominante e detrito foi seu alimento preferencial (Tabela 4). Os demais itens (restos de insetos, Coleoptera e Chironomidae) destacados na figura 4 também foram consumidos por esta espécie em menor quantidade, e foram os alimentos principais de $A$. scabripinnis (Tabela 4).

Em P5 $P$. caudimaculatus e $P$. reticulata foram espécies que, somadas, tiveram o maior número de indivíduos e consumiram detrito como alimento principal. $\mathrm{O}$ item algas filamentosas representado na figura 4 foi o recurso preferencial de T. rendalli. Os itens restos vegetais e restos de insetos foram os alimentos principais de A. scabripinnis, B. iheringii e Hyphessobrycon cf. anisitsi. G. brasiliensis, embora também tenha consumido detrito preferencialmente, junto com $H$. cf. anisitsi, tiveram ainda como importantes os itens Bryozoa e restos orgânicos (Tabela 4). 
Tabela 4. Percentuais dos itens alimentares (em peso) consumidos pelas espécies de peixes dominantes em cada ponto de coleta. Em negrito, os alimentos principais de cada espécies.

\begin{tabular}{|c|c|c|c|c|c|c|c|c|c|c|c|c|c|c|c|c|c|c|c|c|c|}
\hline \multirow[b]{2}{*}{ Recursos } & \multicolumn{2}{|c|}{ Ponto 1} & \multicolumn{5}{|c|}{ Ponto 2} & \multicolumn{5}{|c|}{ Ponto 3} & \multicolumn{2}{|c|}{ Ponto 4} & \multicolumn{7}{|c|}{ Ponto 5} \\
\hline & 2 & 10 & 2 & 5 & 8 & 11 & 15 & 2 & 5 & 8 & 11 & 15 & 2 & 11 & 2 & 3 & 5 & 7 & 10 & 11 & 15 \\
\hline $\begin{array}{l}\text { I. Vegetais } \\
\text { 1) Gramíneas }\end{array}$ & 16,24 & & 1,00 & & & & 11,69 & & & & & 21,78 & & & 0,41 & 0,21 & 1,92 & 0,91 & 0,16 & & \\
\hline 2) Outras sementes & & & & & & & & & & & & & & & & 6,37 & & & & & \\
\hline 3) Plantas Aquáticas & & & & & & & & & & & & & & & 1,52 & 5,68 & 1,98 & 5,14 & 1,74 & 1,66 & 15,18 \\
\hline 4) Algas Filamentosas & & & 0,15 & & & & 2,64 & & & & & 0,10 & & & 1,26 & 0,18 & 0,73 & 0,05 & & & 74,32 \\
\hline 5) Outras Algas & & & & & & & & & & & & & & & 2,04 & & & & & & \\
\hline $\begin{array}{l}\text { 7) Coleoptera } \\
\text { 8) Ephemeroptera }\end{array}$ & $\begin{array}{c}\mathbf{1 4 , 5 6} \\
5,44\end{array}$ & 0,57 & $\begin{array}{c}23,86 \\
0,59\end{array}$ & & & & 0,03 & 8,02 & 0,72 & & & & 24,64 & 0,94 & 2,12 & $\begin{array}{l}0,41 \\
0,63\end{array}$ & & 1,16 & & & \\
\hline 9) Hemiptera & 2,33 & & 10,61 & & & & & 0,07 & & & & 0,54 & 0,86 & & 0,11 & 0,09 & & 0,07 & & & \\
\hline $\begin{array}{l}\text { 10) Hymenoptera } \\
\text { 11) Lepidoptera }\end{array}$ & $\begin{array}{l}\mathbf{9 , 0 5} \\
2,75\end{array}$ & 0,77 & 7,70 & 18,12 & & & & 50,34 & & & 1,64 & & 6,05 & & 9,33 & 4,38 & & 0,43 & & & \\
\hline 12) Odonata & 0,05 & & 7,27 & & & & & & & & & & & & & 0,14 & & 0,33 & & & \\
\hline $\begin{array}{l}\text { 13) Trichoptera } \\
\text { DIPTERA }\end{array}$ & & & & & & & 0,12 & & & & 0,08 & & & & & 0,94 & & & & & \\
\hline 14) Ceratopogonidae & & 0,16 & 0,53 & 3,19 & & 2,99 & 0,35 & 2,14 & 10,30 & 0,07 & 0,45 & 0,09 & 0,80 & & & 0,03 & 0,77 & 0,41 & & 0,76 & 1,07 \\
\hline 15) Chironomidae & 0,13 & 2,04 & 7,68 & 24,91 & 0,04 & 10,54 & & 0,63 & 9,19 & & 2,55 & & 5,44 & 11,00 & 3,86 & 5,90 & 2,90 & 3,61 & 3,75 & 1,19 & \\
\hline 16) Culicidae & 0,87 & & & & & & & & & & & & & & & & & & & & \\
\hline 17) Muscidae & & 1,14 & & & & & & & & & & & 0,88 & & 0,46 & & & & & & \\
\hline 18) Simuliidae & & & 1,99 & 0,38 & & 1,79 & 0,29 & & & & 0,23 & & 0,33 & 2,26 & 0,46 & 0,20 & & & & 0,46 & \\
\hline 19) Outros Diptera & 3,63 & 1,36 & 6,74 & 4,30 & & & 0,04 & 0,61 & 1,78 & & 0,40 & & 3,34 & 2,67 & 7,95 & 5,66 & 0,11 & 1,48 & & & \\
\hline 20) Restos de insetos & 11,54 & 6,57 & 18,90 & 1,60 & & 3,30 & 0,34 & 22,43 & 14,59 & 0,28 & 1,38 & 2,19 & 31,16 & 8,72 & 23,09 & 15,67 & 7,67 & 23,76 & 3,05 & 4,76 & 1,05 \\
\hline III. Crustáceos & & & & & & & & & & & & & & & & & & & & & \\
\hline $\begin{array}{l}\text { 21) Isopoda } \\
\text { 22) Microcrustáceos }\end{array}$ & 2,88 & & & & & & & 0,53 & & & & & & & & & & 0,07 & & & \\
\hline $\begin{array}{l}\text { 22) Microcrustáceos } \\
\text { IV. Peixes }\end{array}$ & & & 5,20 & 0,16 & & & & 0,08 & 1,01 & & & & & 0,24 & 0,33 & & & 0,79 & & 0,23 & 0,02 \\
\hline 23) Escamas e restos & 0,24 & & & & & & 0,68 & & 12,07 & 0,76 & 2,97 & 6,79 & 0,86 & & & 1,19 & & 0,07 & & & \\
\hline $\begin{array}{l}\text { V. Diversos Grupos } \\
\text { 24) Annelida }\end{array}$ & & & & & & & & & & & & & & & & & & & & & \\
\hline $\begin{array}{l}\text { 24) Annelida } \\
\text { 25) Bryozoa }\end{array}$ & & & 0,01 & 0,94 & & & $\begin{array}{l}0,09 \\
0,02\end{array}$ & & $\begin{array}{l}8,76 \\
0,75\end{array}$ & & 0,13 & 7,25 & 1,71 & 3,02 & 5,03 & $\begin{array}{l}1,64 \\
0,17\end{array}$ & 12,66 & 12,38 & 0,76 & 0,21 & $\begin{array}{l}0,29 \\
1,70\end{array}$ \\
\hline 26) Diversos Táxons & 1,48 & 0,24 & 0,57 & & & & & 0,38 & 2,23 & & 0,05 & 0,02 & & & 3,12 & 4,81 & 1,76 & 0,35 & 4,12 & 0,24 & \\
\hline 27) Testacealobosia & 0,08 & 1,07 & & & & & & 0,08 & 0,19 & & & & & & 0,04 & & & & 0,17 & & \\
\hline VI. Outros & & & & & & & & & & & & & & & & & & & & & \\
\hline 28) Detritos & 1,77 & 74,8 & 0,10 & 29,01 & 95,39 & 72,00 & 49,70 & 1,61 & 35,19 & 75,11 & 76,21 & 32,36 & 9,67 & 61,41 & 5,41 & 18,84 & 37,13 & 16,90 & 75,09 & 84,88 & 6,37 \\
\hline 29) Sedimentos* & 2,21 & 10,18 & 0,82 & 11,44 & 4,21 & 9,38 & 1,82 & 0,07 & 3,22 & 18,61 & 9,97 & 5,30 & 2,93 & 9,39 & 0,02 & 0,37 & 0,83 & & 10,25 & 5,22 & \\
\hline 30) Restos orgânicos & & & & & & & 5,70 & & & 2,71 & & & 11,33 & & 3,57 & & 21,89 & 8,31 & & & \\
\hline Total & 100 & 100 & 100 & 100 & 100 & 100 & 100 & 100 & 100 & 100 & 100 & 100 & 100 & 100 & 100 & 100 & 100 & 100 & 100 & 100 & 100 \\
\hline
\end{tabular}

1. Astyanax altiparanae, 2. Astyanax scabripinnis, 3. Bryconamericus iheringii, 4. Callichthys callichthys, 5. Geophagus brasiliensis, 6. Gymnotus carapo, 7. Hyphessobrycon cf. anisitsi, 8. Hypostomus ancistroides, 9. Oreochromis niloticus, 10. Phalloceros caudimaculatus, 11. Poecilia reticulata, 12. Rhamdia quelen, 13. Serrapinnus notomelas, 14. Synbranchus marmoratus e 15. Tilapia rendalli. (*) areia ou torrões de terra, não considerados alimento, mas foram consumidos em quantidades consideráveis pelos exemplares das espécies que comeram no fundo (espécies 5 , 8 , 10 e 11). 


\section{Ponto 1}

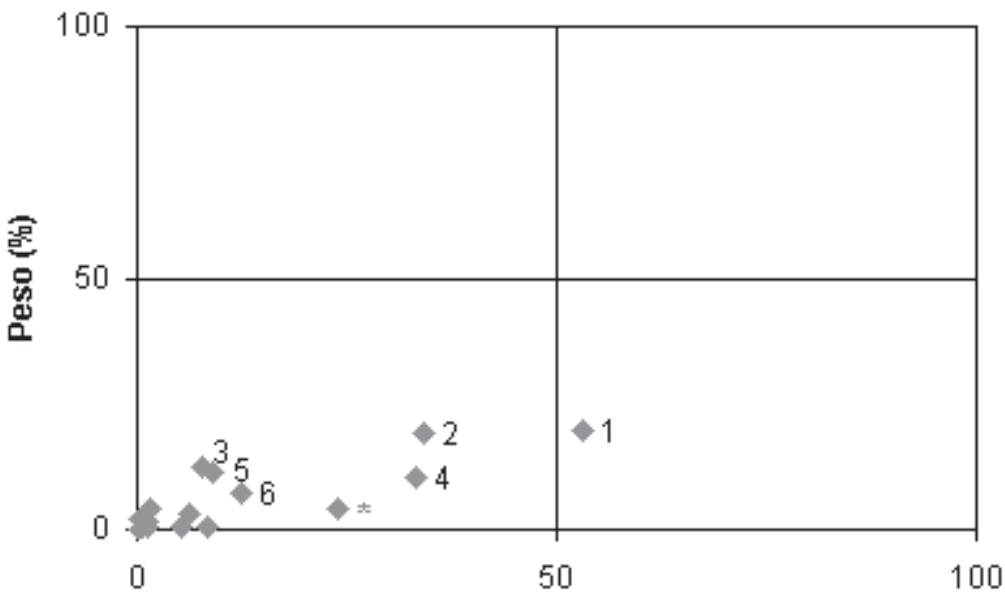

Frequiência de Ocorrência

Ponto 2

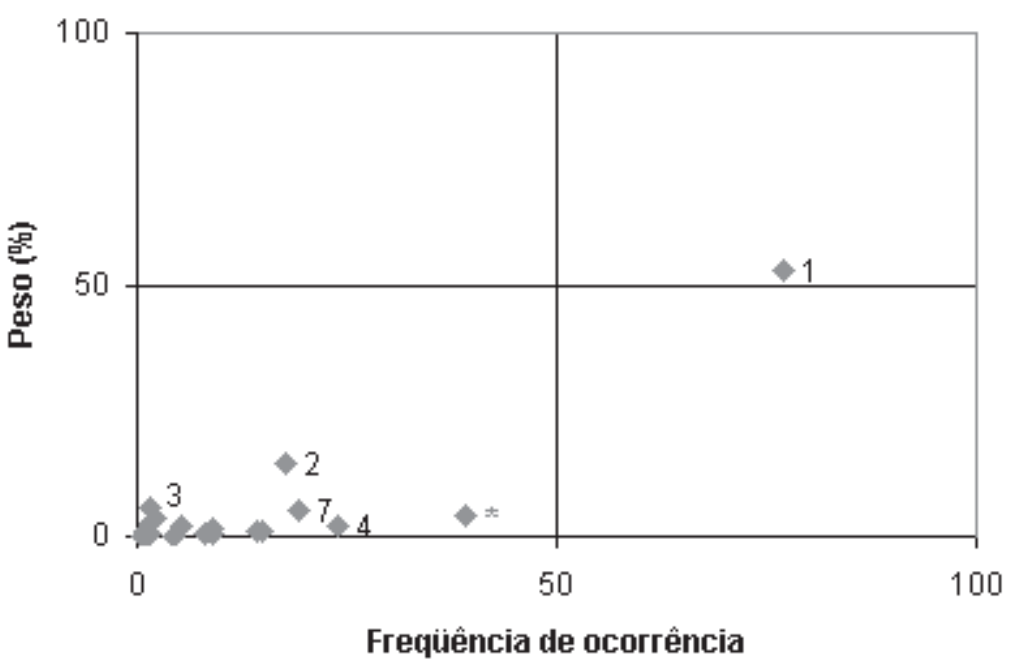

Figura 4. Distribuição dos percentuais de ocorrência e peso dos alimentos consumidos por todas as espécies em cada um dos pontos de coleta (conjugados segundo o método de Costello, 1990). A) ponto 1; B) ponto 2; C) ponto 3; D) ponto 4; E) ponto 5. 1- Detrito, 2- Restos vegetais, 3- Gramíneas, 4- Restos de insetos, 5- Coleoptera, 6- Hymenoptera, 7- Chiromonidae, 8- Bryozoa, 9- Restos Orgânicos, 10- Algas Filamentosas $e^{*}$ - sedimento. 


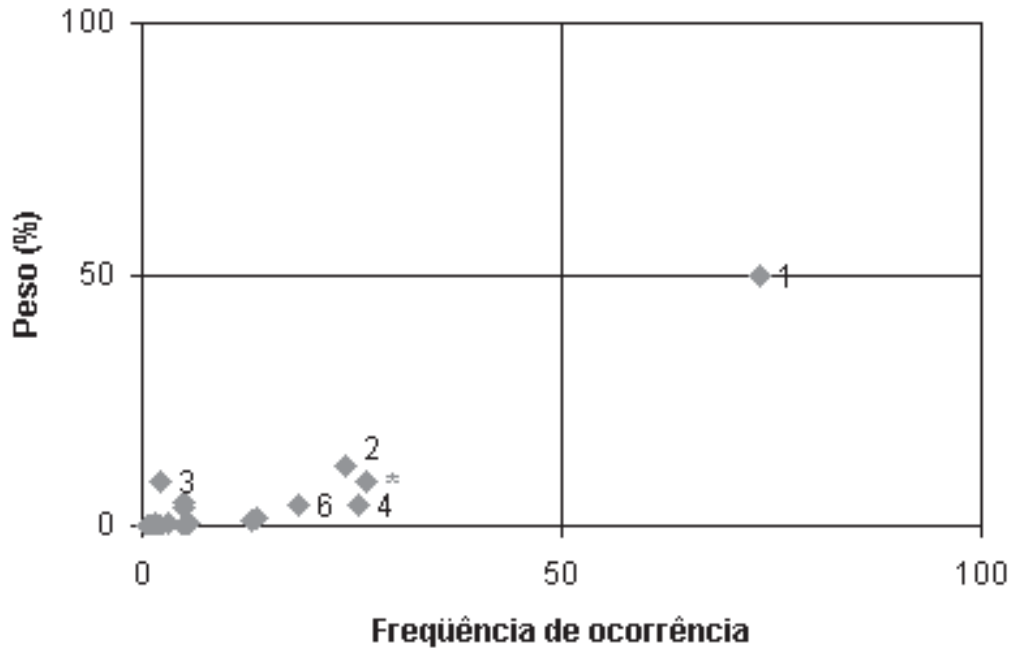

Ponto 4

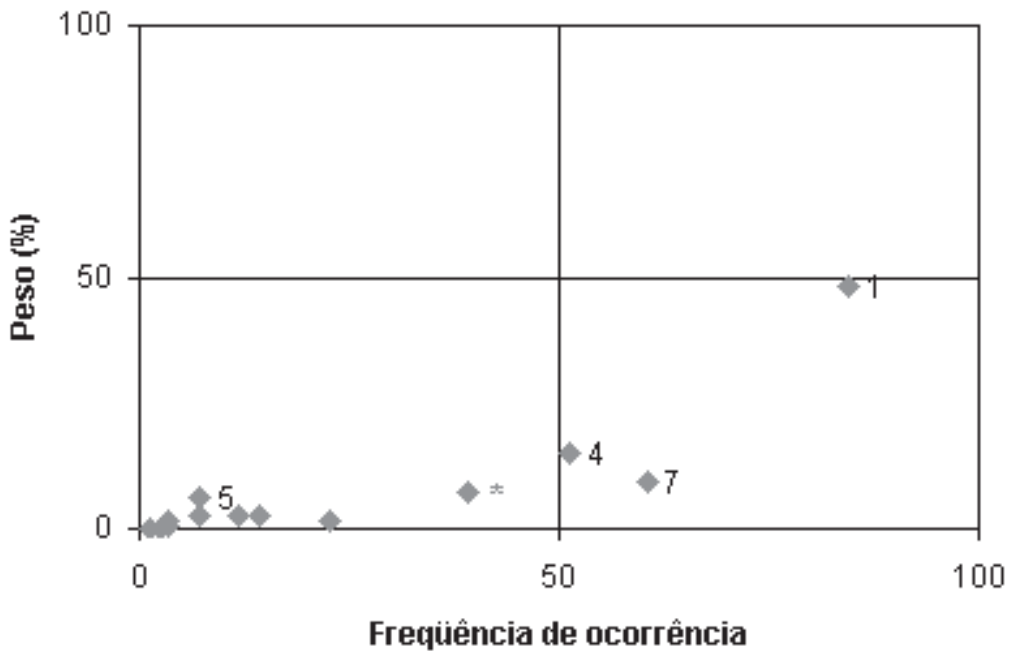

Ponto 5

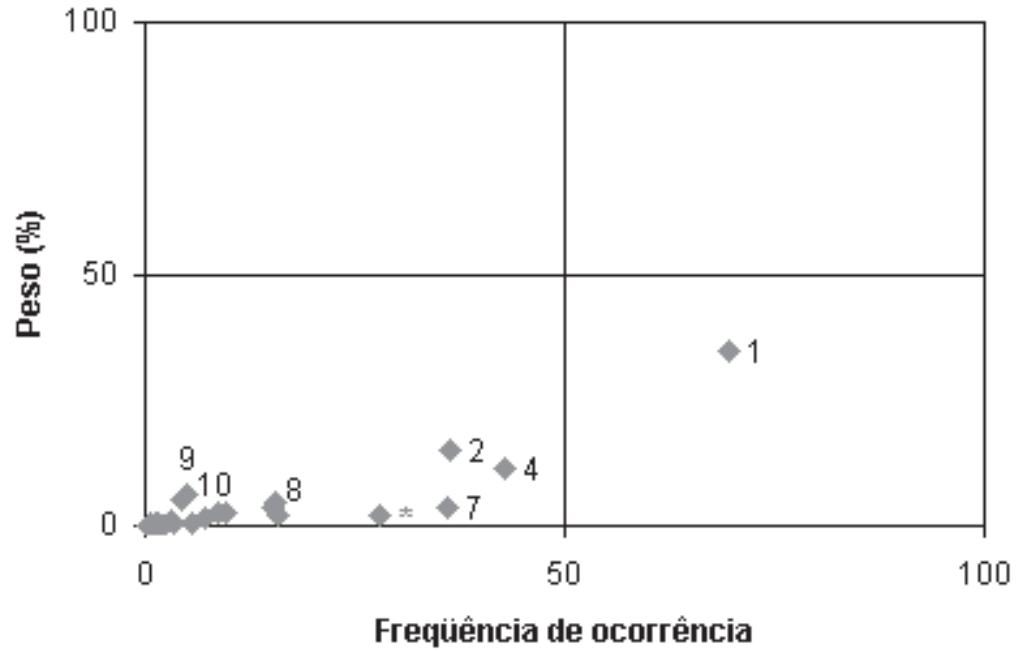




\section{Discussão}

\subsection{Composição da ictiofauna}

Castro (1999) afirma que a predominância de peixes de pequeno porte é o único padrão geral com valor diagnóstico para a ictiofauna de riachos sul-americanos. $\mathrm{O}$ número e a composição das espécies varia muito de acordo com o porte e porção do riacho, região ou bacia. Numa síntese de estudos destes ambientes, Araújo-Lima et al. (1995) verificaram que o número de espécies variou de 7 a 52, e atribuíram esta grande variação em função dos estudos e ambientes comparados terem sido muito heterogêneos. No ribeirão Cambé foram registradas 15 espécies de peixes e, apesar de todos os cinco trechos estarem inseridos na porção superior da drenagem, o número e a composição de espécies foram distintos em cada um dos trechos.

Em porções superiores de riachos da bacia do rio Paraná Abes \& Agostinho (2001) registraram oito espécies, e Casatti (2002) encontrou seis espécies. O maior número de espécies verificado no ribeirão Cambé, se deve em parte à presença de espécies introduzidas. A alta abundância de $P$. reticulata nos pontos $\mathrm{P} 3, \mathrm{P} 4$ e $\mathrm{P} 5$ e de $T$. rendalli em $\mathrm{P} 2$ estão indicando as principais diferenças. No entanto, algumas das espécies encontradas pelos autores acima mencionados não foram encontradas no ribeirão Cambé. Um exemplo disso é o que verificou Casatti (2002), que encontrou uma espécie do gênero Trichomycterus, típico de riachos relativamente pouco degradados. O mesmo foi verificado por Shibatta \& Cheida (2003) em outros riachos da região baixa da bacia do rio Tibagi.

\subsection{Relações entre a composição da ictiofauna, os recursos alimentares consumidos e as interferências antrópicas}

Os peixes do ribeirão Cambé utilizaram uma diversidade alta de itens alimentares, sendo identificados um total de 30. Luiz et al. (1998), estudando dois riachos da bacia do rio Paraná, verificaram que 31 espécies de peixes utilizaram 27 itens alimentares. Vilella et al. (2002), num riacho da bacia do rio Tramandaí no Rio Grande do Sul identificaram 30 itens alimentares utilizados por quatro espécies de Astyanax. Quando os itens são agrupados em categorias, insetos e vegetais terrestres são os recursos de maior destaque, como sumarizado por Araújo-Lima et al. (1995) em vários riachos. Contudo, a alta dominância do detrito utilizado pelas espécies do ribeirão Cambé difere do que é geralmente encontrado em riachos, podendo estar relacionada a alterações ambientais.

De um modo geral, é descrito na literatura que grande parte do que é consumido pelos peixes de riachos é de origem alóctone (Angermeier \& Karr 1984, Sabino \& Castro 1990, Henry et al. 1994, Castro 1999, Esteves \& Aranha 1999, LoweMcConnell 1999). Isto foi verificado em P1 do ribeirão
Cambé, onde as alterações ainda não comprometeram totalmente o ambiente. As alterações foram mais evidentes pela quantidade de detrito (de origem mista), verificada nos demais pontos.

São muitas as relações existentes entre os sistemas terrestres e aquáticos (Barrella et al. 2000). A remoção da mata ciliar pode ser uma das alterações mais danosas a estas relações. A elevada abundância de recursos de origem mista (essencialmente composta por detrito) consumida pelos peixes do ribeirão Cambé pode ser explicada, em parte, por esta alteração. Outros tipos de interferências antrópicas também podem alterar a diversidade tanto de espécies de peixes quanto de recursos alimentares.

Analisando as espécies ao longo da extensão estudada, os itens alimentares consumidos pelos peixes e os impactos antrópicos, foram encontradas situações particulares a cada um dos trechos. Em P1, não se constatou dominância absoluta de um único alimento, pois as espécies mais abundantes (A. scabripinnis e P. caudimaculatus) se comportaram de maneira generalista, e foram responsáveis pelo alto percentual do peso de insetos e vegetais alóctones consumidos. Ambas consideradas onívoras por Castro \& Casatti (1997), são abundantes em águas limpas, não suportando condições de extrema adversidade. No entanto, $P$. caudimaculatus é capaz de sobreviver tanto em ambientes com mata ciliar quanto em ambientes desflorestados (Castro \& Casatti 1997). No ribeirão Cambé a espécie foi abundante em P1 e P5, onde existe vegetação.

Em P2 e P3, P. reticulata, T. rendalli, G. brasiliensis e $H$. ancistroides, espécies consideradas tolerantes segundo Araújo (1998), foram as de maior abundância, sendo que detrito foi o item mais consumido por elas. Em P2, a abundância de indivíduos de $T$. rendalli pode ter relação com as lagoas artificiais localizadas a montante do ponto de coleta, onde provavelmente foram introduzidos. Em P3, a espécie $P$. reticulata foi a de maior representatividade, e as más condições encontradas no trecho devem ser conseqüência da remoção da mata ciliar e do constante trânsito de pessoas e animais domésticos. A espécie $H$. ancistroides, embora tenha sido considerada herbívora em outros estudos (Castro \& Casatti 1997 e Esteves \& LobónCerviá 2001), nos trechos do ribeirão Cambé se comportou essencialmente como detritívora. Resultado similar foi encontrado para G. brasiliensis, uma espécie considerada onívora em ambientes relativamente preservados (Sabino \& Castro 1990). A. scabripinnis, sensível a alterações ambientais, foi constatada em baixo número de exemplares.

O ponto P4 foi o trecho que apresentou a vegetação ripária com melhores condições de preservação, porém a diversidade de espécies e de itens alimentares utilizados pelos peixes foi a menor. É provável que a qualidade da água seja o fator limitante para a ocorrência de poucas espécies e de poucos itens alimentares, pois à montante do 
trecho são escoados resíduos de uma indústria de empacotamento de leite. Neste ponto, apenas $P$. reticulata foi dominante por ser capaz de sobreviver até mesmo em águas contaminadas por metais pesados (Araújo 1998, Lemes \& Garutti 2002). Por se tratar de uma espécie oportunista, utilizou detrito como alimento principal, possivelmente por ser muito abundante no trecho, e não os recursos de origem alóctone proveniente da mata (insetos e partes de vegetais).

Em P5, apesar de ter parte das características originais de seu leito alteradas pela formação de um lago artificial, foi registrada a maior diversidade de itens alimentares e o maior número de espécies. Além disso, neste trecho as proporções de vegetal, animal e detrito estiveram mais próximas da eqüitabilidade. As alterações nas características físicas do leito foram as responsáveis pela maior heterogeneidade do hábitat, promovendo um aumento na disponibilidade de micro-hábitats, que diretamente influencia a distribuição das espécies (Barreto \& Uieda 1998). Apesar desta diversidade de habitats, o detrito continuou em alta disponibilidade, favorecendo $P$. reticulata (44\% do total coletado no trecho), que juntamente com $P$. caudimaculatus foram as principais responsáveis pela quantidade de detrito consumido no trecho. Os demais itens registrados foram consumidos pelas quatro espécies de Tetragonopterinae (A. scabripinnis, Astyanax altiparanae, B. iheringii e $H$. cf. anisitsi).

Em síntese, foi possível perceber que as diferentes ações antrópicas num ambiente urbano são responsáveis por alterações não só na composição da ictiofauna, como também na dieta dos peixes. No ribeirão Cambé ficou bem evidente o efeito de quatro categorias de impactos: remoção da mata ciliar, lançamento de efluentes na água, introdução de espécies e alterações físicas no leito do riacho. No caso de grandes impactos no ambiente, a tendência é que permaneçam as espécies mais tolerantes e a exóticas passem a representar a maioria dos indivíduos da ictiofauna (Lyons et al. 1995). Tal cenário se ajusta perfeitamente ao ribeirão Cambé, onde $P$. reticulata dominou em número de indivíduos nos pontos mais impactados. Além disso, esta espécie demonstrou grande oportunismo em utilizar um item dominante (detrito) e não uma variedade de itens como seria esperado em ambientes menos alterados.

\section{Agradecimentos}

Agradecemos a Dr. Oscar A. Shibatta pela identificação das espécies de peixes; ao convênio UEL/ FAUEL/CONFEPAR pelo apoio financeiro; à CAPES pela bolsa concedida; à Dra Norma Segatti Hahn e Dr. Reinaldo José de Castro pelas valiosas sugestões e correções da Dissertação de Mestrado que deu origem a esta pesquisa; ao Dr. Luiz dos Anjos pela leitura prévia dos manuscritos e aos técnicos do Museu de Zoologia/MZUEL, Edson Santana da Silva e Aparecido de Souza.

\section{Referências Bibliográficas}

ABES, S. DA S. \& AGOSTINHO, A.A. 2001. Spatial patterns in fish distributions and structure of the ichthyocenosis in the Água Nanci stream, upper Paraná River basin, Brazil. Hydrobiologia 445:217-227.

ANGERMEIER, P.L. \& KARR, J.R. 1984. Fish communities in a system of tropical streams. In Evolutionary ecology of neotropical freshwater fishes (T.M. Zaret, ed.). Dr. W. Junk Publishers. The Hague, Netherlands, p.39-57.

ARAÚJO-LIMA, C.A.R.M., AGOSTINHO, A.A. \& FABRÉ, N.N. 1995. Trophic aspects of fish communities in Brazilian rivers and reservoirs. In Limnology in Brazil (J.G. Tundisi, C.E.M. Bicudo \& T. Matsumura-Tundisi, eds.). ABC/SBL, Rio de Janeiro, p.105-136.

ARAÚJO, F.G. 1998. Adaptação do índice de integridade biótica usando a comunidade de peixes para o rio Paraíba do Sul. Rev. Brasil. Biol. 58(4):547-558.

BARRELLA, W., PETRERE JR., M., SMITH, W.S. \& MONTAG, L.F.A. 2000. As relações entre as matas ciliares, os rios e os peixes. In Matas ciliares: Conservação e recuperação (R.R. Rodrigues \& H. F. L. Filho, eds.). EDUSP, São Paulo, p.187-207.

BARRETO, M.G. \& UIEDA, V.S. 1998. Influence of the abiotic factors on the ichthyofauna composition in different orders stretches of Capivara River, São Paulo State, Brazil. Verh. Internat. Verein. Limnol. 26:2180-2183.

CASATTI, L. 2002. Alimentação dos peixes em um riacho do Parque Estadual Morro do Diabo, bacia do alto rio Paraná, sudeste do Brasil. Biota Neotropica 2(2): http:// w w w. bi ot a n e tropica.org.br/v 2 n $2 / \mathrm{pt} /$ download?article+BN02502022002+item

CASTRO, R.M.C. \& CASATTI, L. 1997. The fish fauna from a small forest stream of the upper Paraná river basin, southeastern Brazil. Ichthyol. Explor. Freshwaters 7(4):337-352.

CASTRO, R.M.C. 1999. Evolução da ictiofauna de riachos sul-americanos: padrões gerais e possíveis processos causais. In Ecologia de peixes de riachos (E.P. Caramaschi, R. Mazzoni \& P.R. Peres-Neto, eds.). Série Oecologia Brasiliensis, PPGE-UFRJ, Rio de Janeiro, v.VI, p.139-155.

COSTELLO, M.J. 1990. Predator feeding strategy and prey importance: a new graphical analysis. J. Fish Biol. 36:261263.

ESTEVES, K.E. \& ARANHA, J.M.R. 1999. Ecologia trófica de peixes de riachos. In Ecologia de peixes de riachos (E.P. Caramaschi, R. Mazzoni \& P.R. Peres-Neto, eds.). Série Oecologia Brasiliensis, PPGE-UFRJ, Rio de Janeiro, v. VI, p.157-182. 
ESTEVES, K. \& LOBÓN-CERVIÁ, J. 2001. Composition and trophic structure of a fish community of a clear water Atlantic rainforest stream in southeastern Brazil. Envir. Biol. Fishes 62:429-440.

HENRY, R., UIEDA, V.S., AFONSO, A.A.O. \& KIKUCHI, R.M. 1994. Input of allochthonous matter and structure of fauna in a brazilian headstream. Verh. Internat. Verein. Limnol. 25(3):1866-1870.

HYSLOP, J. 1980. Stomach contents analysis - a review of methods and their application. J. Fish Biol. 17:411-429.

LEMES, E.M. \& GARUTTI, V. 2002. Ecologia da ictiofauna de um córrego de cabeceira da bacia do alto rio Paraná, Brasil. Iheringia, Ser. Zool. 92(3):69-78.

LOWE-McCONNELL, R.H. 1999. Estudos ecológicos de comunidades de peixes tropicais. (A.E.A.M. Vazzoler, A.A. Agostinho \& P.T.M. Cunnhingham, trad.). EDUSP, São Paulo.

LUIZ, E.A., AGOSTINHO, A. A., GOMES, L.C. \& HAHN, N.S. 1998. Ecologia trófica de peixes em dois riachos da bacia do rio Paraná. Rev. Bras. Biol. 58(2):273-285.

LYONS, J., NAVARRO-PÉREZ, S., COCHRAN, P.A., SANTANA, C. \& GUZMÁN-ARROYO, M. 1995. Index of biotic integrity based on fish assemblages for the conservation of streams and rivers in west-central México. Cons. Biol. 9(3):569-584.

PÉREZ, G.R. 1998. Guia para el estudio de los macroinvertebrados acuáticos del Departamento de Antioquia. Bogotá.

SABINO, J. \& CASTRO, R.M.C. 1990. Alimentação, período de atividade e distribuição espacial dos peixes de um riacho da Floresta Atlântica (Sudeste do Brasil). Rev. Brasil. Biol. 50(1):23-36.

SHIBATTA, O.A., ORSI, M.L., BENNEMANN, S.T. \& SILVA-SOUZA, Â.T. 2002. Diversidade e distribuição de peixes na bacia do rio Tibagi. In A bacia do rio Tibagi (M.E. Medri, E. Bianchini, O.A. Shibatta \& J.A. Pimenta, eds.). Londrina, p.403-423.

SHIBATTA, O.A. \& CHEIDA, C.C. 2003. Composição em tamanho dos peixes (Actinopterygii, Teleostei) de ribeirões da bacia do rio Tibagi, Paraná, Brasil. Revta. brasil. Zool. 20(3):469-473.

STEHR, F.W. 1987. Imature Insects. Kendal/Hunt Publishing Company, Iowa.

VEREGUE, A.M.L. \& ORSI, M.L. 2003. Biologia reprodutiva de Astyanax scabripinnis paranae (Eigenmann) (Osteichthyes, Characidae), do ribeirão das Marrecas, bacia do rio Tibagi, Paraná. Revta. brasil. Zool. 20(1):97-105.

VILELLA, F.S., BECKER, F.G. \& HARTS, S.M. 2002. Diet of Astyanax species (Teleostei, Characidae) in an Atlantic Forest River in Southern Brazil. Braz. arch. biol. technol., 45(2):223-232.
Título: Ictiofauna, recursos alimentares e relações com as interferências antrópicas em um riacho urbano no sul do Brasil.

Autores: Deise Cristiane de Oliveira \& Sirlei Terezinha Bennemann

Biota Neotropica, Vol. 5 ( número 1): 2005

http://www.biotaneotropica.org.br/v5n1/pt/ abstract?article+BN02905012005

Recebido em 01/09/04

Versão revisada recebida em 20/01/05

Publicado em 01/02/05

ISSN 1676-0603 